\title{
Self-synchronization and Automatic Balancing in Rotor Dynamics*
}

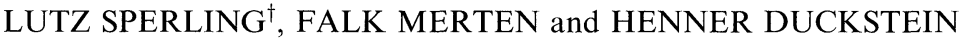 \\ Otto-von-Guericke-Universität Magdeburg, PF 4120, 39016 Magdeburg, Germany
}

(Received 12 May 1998; In final form 10 October 1998)

\begin{abstract}
Synchronous elimination as one of the possible methods of cancelling any harmful vibration resulting from the unbalance of rotary machines is considered. This method, introduced by Fesca and Thearle, involves the placement of unbalanced elements (e.g. ring, pendulum, ball balancers) on the rotor axis, which can occupy any angular position in relation to the rotor. Under defined conditions in the postcritical frequency range, there is a spontaneous placement of the corection elements such that they balance the rotor unbalance. Hedaya and Sharp generalized this method by combining two force balancers to compensate the unbalanced moment as well as the unbalanced force of a rigid rotor.

In the present work this generalization is analysed in detail. The existence and the stability conditions of compensating phasing are developed by the method of direct motion separation, the dependence of these conditions on the polar inertia is investigated, and the validity of Blekhman's principle of self-balancing is discussed.

The results are confirmed by computer simulation. Moreover, computer simulation is used to investigate transient motions induced by initial conditions.
\end{abstract}

Keywords: Automatic dynamic balancers, Synchronous elimination, Generalized self-balancing principle, Analytical approach, Stability condition, Computer simulation

\section{INTRODUCTION}

Thearle and Schenectady (1932) proposed a balancing machine for rigid rotors which employs two spherical balls being free to move in a viscous medium along a circular track fixed to the rotor. Under certain conditions the balls in the high frequency range seek positions such as to counter any unbalance present in the rotor itself.

Hedaya and Sharp (1977) generalized this device introducing a new type of dynamic balancer which combines two force balancers separated by an appropriate distance so that they act together to compensate the unbalanced moment as well as the

\footnotetext{
* This paper was originally presented at ISROMAC-7.

${ }^{\dagger}$ Corresponding author. Tel.: (0391) 67-12 440. Fax: (0391) 67-12 439. E-mail: lutz.sperling@mb.uni.magdeburg.de
} 
unbalanced force. A stability analysis of the balanced condition together with results of parametric studies is presented. It is referred to experimental and simulation study, but without any report on the results.

A similar problem was investigated by Sperling et al. (1997), where the oscillating system does not rotate and the forces and moments which are to be compensated are caused by two circulating mass points. The compensation is realized by separately driven circulating mass points. This is a typical example of self-synchronization of unbalanced rotors.

A well suited method for the derivation of the conditions for the existence and the stability of synchronous motion applying harmonic influence coefficients is described in Sperling (1994). It is based upon the general theory of synchronization explained in detail by Blekhman (1988). In the case of synchronous motion the kinetic energy of the oscillating system under certain conditions is minimized in the frequency range above the highest natural frequency. From this follows the stability of any fully compensating motion if such motion exists. In generalization of this phenomenon Blekhman (1993) formulated a generalized (rotors) self-balancing principle. An important condition for the validity of this principle is the independence of the eigenfrequencies from the angular velocity.

The goal of the present paper focuses on further investigations of the problem of Hedaya and Sharp. If the rotor is a rigid thin bar the only difference in comparison to the problem investigated in Sperling et al. (1997) is that the constant terms of the driving moments acting on the compensating rotors are replaced by the moments due to the viscous medium. In this case the results of computer simulation show the same principal behaviour as in the case of separately driven unbalanced rotors on a non-rotating oscillating system. However, if the polar inertia of the basic rotor is not negligible, the eigenfrequencies depend upon the angular velocity. Hence the compensation solutions can be unstable in the high frequency range also.
Therefore, in the present paper the approximate analytic investigation is accomplished by means of the method of direct motion separation (Kapiza, 1951), a simple special version of the method of multiple scales, well suited for the investigation of the self-synchronization of separately driven rotors on a non-rotating system as well (Blekhman, 1976; Sperling et al., 1997). In contradistinction from the paper of Hedaya and Sharp (1977) the balls are idealized in a simpler manner as point masses and we give only qualitative statements concerning the stability with respect to the parameter values. Nevertheless, some results can be formulated in an explicit form.

Moreover, the paper contains some results of diverse computer simulations confirming the results of the analytic investigations and giving, in addition, an insight into transient motions.

\section{MODEL WITH TWO FORCE BALANCERS FOR AUTOMATIC BALANCING OF AN UNBALANCED FORCE AND AN UNBALANCED MOMENT}

The following investigations are devoted to the analysis of automatic balancing of a primary statically and dynamically unbalanced rigid rotor by means of two simplified Thearle type force balancers (Fig. 1).

Before explaining this system in detail, a rigid massless axis A with four degrees of freedom, corresponding to the generalized coordinates

$$
\boldsymbol{q}=\left[x ; y ; \psi_{x} ; \psi_{y}\right]^{\mathrm{T}}
$$

is considered (Fig. 2).

First we assume that only one general rigid body rotor with arbitrary mass distribution and with one relative degree of freedom, corresponding to angle $\varphi$, is mounted on the axis. $m$ is the mass of the rotor,

$$
\boldsymbol{J}=\left[\begin{array}{lll}
J_{\mathrm{rr}} & J_{\mathrm{rs}} & J_{\mathrm{r} z} \\
J_{\mathrm{sr}} & J_{\mathrm{ss}} & J_{\mathrm{s} z} \\
J_{z \mathrm{r}} & J_{z \mathrm{~s}} & J_{z z}
\end{array}\right]
$$




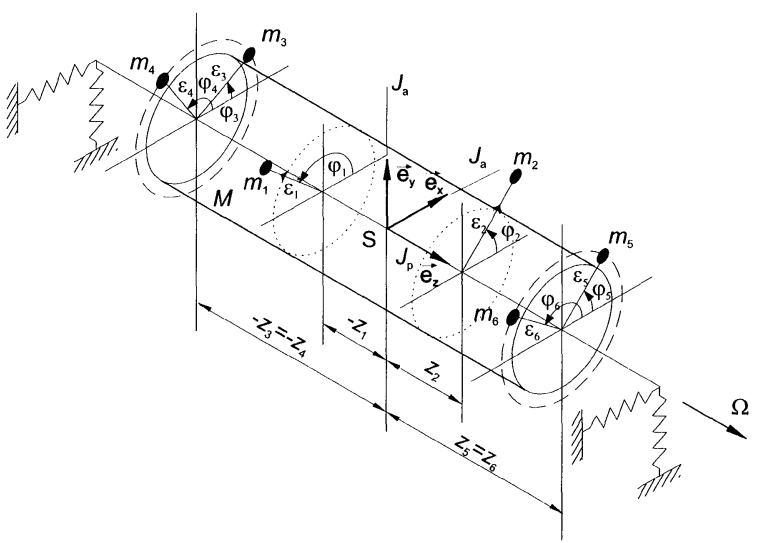

FIGURE 1 Unbalanced rigid body rotor with two force balancers.
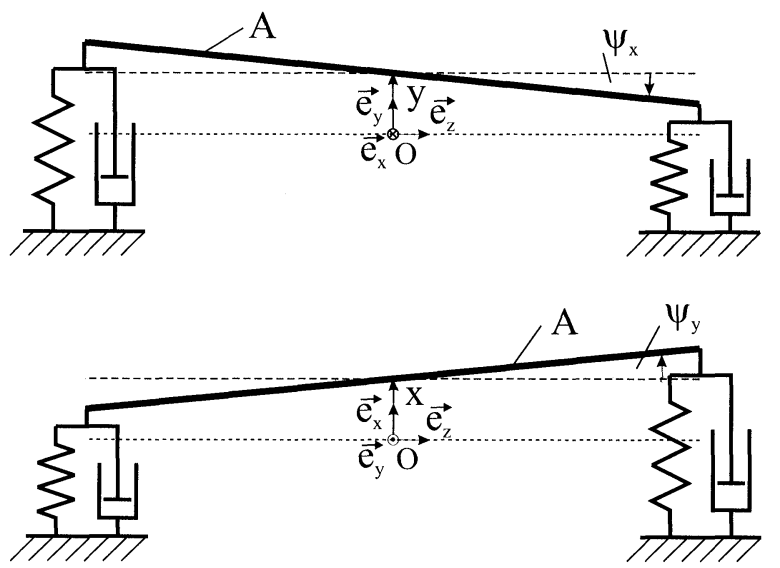

FIGURE 2 Rigid massless axis with four degrees of freedom.

is its matrix of the moments and products of inertia with respect to the mass centre $\mathrm{C}$ and the vector basis $\overrightarrow{\boldsymbol{e}}_{\mathrm{r}}, \overrightarrow{\boldsymbol{e}}_{\mathrm{s}}, \overrightarrow{\boldsymbol{e}}_{z}$ (Fig. 3).

The pivot point $\mathrm{O}_{z}$, i.e. the point of intersection of axis $z$ and the plane of the motion of the point $\mathrm{C}$, has the coordinate $z$ as the distance between $\mathrm{O}$ and $\mathrm{O}_{z}$.

Now we can explain our system according to Fig. 1 as seven special rigid body rotors, mounted on the axis $\mathrm{A}$.

The primary rotor consists of a balanced symmetric basic rotor (marked by index 0 ) with its

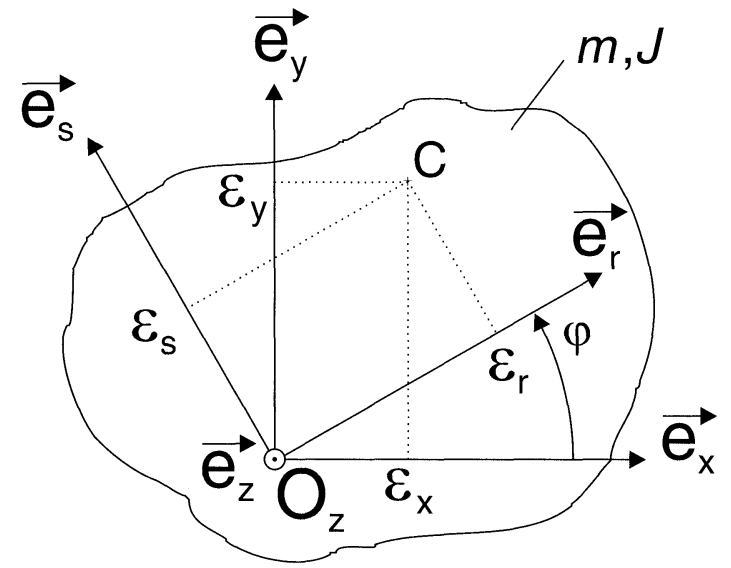

FIGURE 3 General rigid body rotor with one relative degree of freedom.

mass centre $\mathrm{C}_{0}$ in $\mathrm{O}$, i.e.

$$
z_{0}=0
$$

and with

$$
\begin{gathered}
m_{0}=M, \quad \varepsilon_{\mathrm{r} 0}=0, \quad \varepsilon_{\mathrm{s} 0}=0, \\
\boldsymbol{J}_{0}=\left[\begin{array}{ccc}
J_{\mathrm{a}} & 0 & 0 \\
0 & J_{\mathrm{a}} & 0 \\
0 & 0 & J_{\mathrm{p}}
\end{array}\right]
\end{gathered}
$$

and of two point masses, indices 1 and 2, rigidly attached to the basic rotor and representing the unbalance of the primary rotor. The force balancers contain four point masses, number 3-6. For all point masses it is proposed

$$
\boldsymbol{J}_{i}=\mathbf{0}, \varepsilon_{\mathrm{s} i}=0, \varepsilon_{\mathrm{r} i}=\varepsilon_{i}, \quad i=1, \ldots, 6
$$

We define the angle $\varphi_{0}$ by the position of the unbalance number 1 and the phase between the unbalances number 1 and 2 by the angle $\gamma$ :

$$
\varphi_{1}=\varphi_{0}, \quad \varphi_{2}=\varphi_{0}+\gamma
$$

The masses and unbalances of the compensating point masses, arranged in two forces balancers with 
distances $z_{3}$ and $z_{5}$ with respect to $\mathrm{O}$ and freely movable in a viscous medium, are assumed to be equal in pairs,

$$
\begin{array}{cl}
m_{4}=m_{3}, & m_{6}=m_{5}, \quad \varepsilon_{4}=\varepsilon_{3}, \quad \varepsilon_{6}=\varepsilon_{5}, \\
& z_{4}=z_{3}, \quad z_{6}=z_{5} .
\end{array}
$$

\section{EQUATIONS OF MOTION}

The equations of motion of the axis A system with only one general rigid body rotor according to Figs. 2 and 3 are

$$
\boldsymbol{Q}+\boldsymbol{C} \cdot \dot{\boldsymbol{q}}+\boldsymbol{K} \cdot \boldsymbol{q}=\mathbf{0}
$$

$\boldsymbol{C}$ and $\boldsymbol{K}$ are the damping and stiffness matrices, and

$$
\boldsymbol{Q}=\left[Q_{x} ; Q_{y} ; Q_{\psi x}-z Q_{y} ; Q_{\psi y}+z Q_{x}\right]^{\mathrm{T}}
$$

is the column matrix of all types of acceleration terms, linearized in $\boldsymbol{q}$.

The development of the $Q$ terms is an expensive standard problem of analytical dynamics. We remark that it is realized in detail for a more general case in Sperling (1994). Therefore, we confine to present the following result:

$$
\begin{aligned}
Q_{x}= & m\left[\ddot{x}+z \ddot{\psi}_{y}-\varepsilon_{x} \dot{\varphi}^{2}-\varepsilon_{y} \ddot{\varphi}\right], \\
Q_{y}= & m\left[\ddot{y}-z \ddot{\psi}_{x}-\varepsilon_{y} \dot{\varphi}^{2}+\varepsilon_{x} \ddot{\varphi}\right], \\
Q_{\psi x}= & \bar{J}_{x x} \ddot{\psi}_{x}+\bar{J}_{x y} \ddot{\psi}_{y}+\left[-2 \bar{J}_{x y} \dot{\psi}_{x}\right. \\
& \left.+\left(\bar{J}_{x x}-\bar{J}_{y y}+\bar{J}_{z z}\right) \dot{\psi}_{y}\right] \dot{\varphi} \\
& -J_{y z} \dot{\varphi}^{2}+\left(J_{x z}+\frac{1}{2} \bar{J}_{z z} \psi_{y}\right) \ddot{\varphi}, \\
Q_{\psi y}= & \bar{J}_{x y} \ddot{\psi}_{x}+\bar{J}_{y y} \ddot{\psi}_{y} \\
& +\left[\left(\bar{J}_{x x}-\bar{J}_{y y}-\bar{J}_{z z}\right) \dot{\psi}_{x}+2 \bar{J}_{x y} \dot{\psi}_{y}\right] \dot{\varphi} \\
& +J_{x z} \dot{\varphi}^{2}+\left(J_{y z}-\frac{1}{2} \bar{J}_{z z} \psi_{x}\right) \ddot{\varphi},
\end{aligned}
$$

with

$$
\varepsilon_{x}^{2}=\frac{1}{2}\left(\varepsilon_{\mathrm{r}}^{2}+\varepsilon_{\mathrm{s}}^{2}\right)+\frac{1}{2}\left(\varepsilon_{\mathrm{r}}^{2}-\varepsilon_{\mathrm{s}}^{2}\right) \cos 2 \varphi-\varepsilon_{\mathrm{r}} \varepsilon_{\mathrm{s}} \sin 2 \varphi,
$$

$$
\begin{aligned}
\varepsilon_{y}^{2} & =\frac{1}{2}\left(\varepsilon_{\mathrm{r}}^{2}+\varepsilon_{\mathrm{s}}^{2}\right)-\frac{1}{2}\left(\varepsilon_{\mathrm{r}}^{2}-\varepsilon_{\mathrm{s}}^{2}\right) \cos 2 \varphi+\varepsilon_{\mathrm{r}} \varepsilon_{\mathrm{s}} \sin 2 \varphi, \\
\varepsilon_{x} \varepsilon_{y} & =\frac{1}{2}\left(\varepsilon_{\mathrm{r}}^{2}-\varepsilon_{\mathrm{s}}^{2}\right) \sin 2 \varphi+\varepsilon_{\mathrm{r}} \varepsilon_{\mathrm{s}} \cos 2 \varphi, \\
J_{x x} & =\frac{1}{2}\left(J_{\mathrm{rr}}+J_{\mathrm{ss}}\right)+\frac{1}{2}\left(J_{\mathrm{rr}}-J_{\mathrm{ss}}\right) \cos 2 \varphi-J_{\mathrm{rs}} \sin 2 \varphi,
\end{aligned}
$$

$J_{y y}=\frac{1}{2}\left(J_{\mathrm{rr}}+J_{\mathrm{ss}}\right)-\frac{1}{2}\left(J_{\mathrm{rr}}-J_{\mathrm{ss}}\right) \cos 2 \varphi+J_{\mathrm{rs}} \sin 2 \varphi$,

$$
J_{x y}=\frac{1}{2}\left(J_{\mathrm{rr}}-J_{\mathrm{ss}}\right) \sin 2 \varphi+J_{\mathrm{rs}} \cos 2 \varphi,
$$

$J_{x z}=J_{\mathrm{r} z} \cos \varphi-J_{\mathrm{sz}} \sin \varphi$,

$J_{y z}=J_{\mathrm{r} z} \sin \varphi+J_{\mathrm{sz}} \cos \varphi$,

$\bar{J}_{x x}=J_{x x}+m \varepsilon_{y}^{2}, \quad \bar{J}_{y y}=J_{y y}+m \varepsilon_{x}^{2}$,

$\bar{J}_{x y}=J_{x y}-m \varepsilon_{x} \varepsilon_{y}$,

$\bar{J}_{z z}=J_{z z}+m\left(\varepsilon_{\mathrm{r}}^{2}+\varepsilon_{\mathrm{s}}^{2}\right)$.

The equation of motion of the rigid body rotor, linearized with respect to $\boldsymbol{q}$ as well (see Sperling, 1994) is

$$
\bar{J}_{z z} \ddot{\varphi}+B=Q_{\varphi},
$$

with

$$
\begin{aligned}
B= & \left(-m \varepsilon_{\mathrm{s}} \ddot{x}+m \varepsilon_{\mathrm{r}} \ddot{y}+\bar{J}_{\mathrm{r} z} \ddot{\psi}_{x}+\bar{J}_{\mathrm{s} z} \ddot{\psi}_{y}\right) \cos \varphi \\
& -\left(m \varepsilon_{\mathrm{r}} \ddot{x}+m \varepsilon_{\mathrm{s}} \ddot{y}+\bar{J}_{\mathrm{s} z} \ddot{\psi}_{x}-\bar{J}_{\mathrm{r} z} \ddot{\psi}_{y}\right) \sin \varphi, \\
\bar{J}_{\mathrm{r} z}= & J_{\mathrm{r} z}-m \varepsilon_{\mathrm{r}} z, \quad \bar{J}_{\mathrm{s} z}=J_{\mathrm{s} z}-m \varepsilon_{\mathrm{s}} z,
\end{aligned}
$$

$Q_{\varphi}$ being the driving and damping torque.

From the equations of motion (10) it follows for the system according to Fig. 1 that

$$
\boldsymbol{Q}_{\text {total }}+\boldsymbol{C} \cdot \dot{\boldsymbol{q}}+\boldsymbol{K} \cdot \boldsymbol{q}=\mathbf{0},
$$


with the column matrix of the sum of all acceleration terms

$$
\begin{gathered}
\boldsymbol{Q}_{\text {total }}=\sum_{i=0}^{6} \boldsymbol{Q}_{i}=\sum_{i=0}^{6}[ \\
{\left[Q_{x i} ; Q_{y i} ; Q_{\psi x i}-z_{i} Q_{y i} ; Q_{\psi y i}\right.} \\
\left.+z_{i} Q_{\psi x i}\right]^{\mathrm{T}} .
\end{gathered}
$$

The $Q$ terms can easily be determined as special cases of expressions (12)-(15).

Thus, we find for the basic rotor

$$
\begin{aligned}
Q_{x 0} & =M \ddot{x}, \quad Q_{y o}=M \ddot{y}, \\
Q_{\psi x 0} & =J_{\mathrm{a}} \ddot{\psi}_{x}+J_{\mathrm{p}} \dot{\varphi}_{0} \dot{\psi}_{y}+\frac{1}{2} J_{\mathrm{p}} \ddot{\varphi}_{0} \psi_{y}, \\
Q_{\psi y 0} & =J_{\mathrm{a}} \ddot{\psi}_{y}-J_{\mathrm{p}} \dot{\varphi}_{0} \dot{\psi}_{x}-\frac{1}{2} J_{\mathrm{p}} \ddot{\varphi}_{0} \psi_{x} .
\end{aligned}
$$

The $Q$ terms of all point masses are

$$
\begin{aligned}
Q_{x i}= & m_{i}\left[\ddot{x}+z_{i} \ddot{\psi}_{y}-\varepsilon_{i}\left(\dot{\varphi}_{i}^{2} \cos \varphi_{i}+\ddot{\varphi}_{i} \sin \varphi_{i}\right)\right], \\
Q_{y i}= & m_{i}\left[\ddot{y}-z_{i} \ddot{\psi}_{x}-\varepsilon_{i}\left(\dot{\varphi}_{i}^{2} \sin \varphi_{i}-\ddot{\varphi}_{i} \cos \varphi_{i}\right)\right], \\
Q_{\psi x i}= & m_{i} \varepsilon_{i}^{2}\left\{\left[\left(\ddot{\psi}_{x}+2 \dot{\varphi}_{i} \dot{\psi}_{y}\right) \sin \varphi_{i}\right.\right. \\
& \left.\left.-\left(\ddot{\psi}_{y}-2 \dot{\varphi}_{i} \dot{\psi}_{x}\right) \cos \varphi_{i}\right] \sin \varphi_{i}+\frac{1}{2} \ddot{\varphi}_{i} \psi_{y}\right\}, \\
Q_{\psi y i}= & -m_{i} \varepsilon_{i}^{2}\left\{\left[\left(\ddot{\psi}_{x}+2 \dot{\varphi}_{i} \dot{\psi}_{y}\right) \sin \varphi_{i}\right.\right. \\
& \left.\left.-\left(\ddot{\psi}_{y}-2 \dot{\varphi}_{i} \dot{\psi}_{x}\right) \cos \varphi_{i}\right] \cos \varphi_{i}+\frac{1}{2} \ddot{\varphi}_{i} \psi_{x}\right\},
\end{aligned}
$$

with $i=1, \ldots, 6$. Equation (25) yields the equation of motion of the primary rotor

$$
\begin{aligned}
\left(J_{\mathrm{p}}\right. & \left.+m_{1} \varepsilon_{1}^{2}+m_{2} \varepsilon_{2}^{2}\right) \ddot{\varphi}_{0}+B_{0} \\
& =L_{0}\left(\dot{\varphi}_{0}\right)-\sum_{i=3}^{6} \beta_{i}\left(\dot{\varphi}_{0}-\dot{\varphi}_{i}\right) \\
& =L_{0}^{0}-\beta_{0} \dot{\varphi}_{0}+\sum_{i=3}^{6} \beta_{i} \dot{\varphi}_{i},
\end{aligned}
$$

with

$B_{0}=\sum_{i=0}^{2} m_{i} \varepsilon_{i}\left[-\left(\ddot{x}+z_{i} \ddot{\psi}_{y}\right) \sin \varphi_{i}+\left(\ddot{y}-z_{i} \ddot{\psi}_{x}\right) \cos \varphi_{i}\right]$ and the equations of motion of the compensating point masses

$$
m_{i} \varepsilon_{i}^{2} \ddot{\varphi}_{i}+B_{i}=\beta_{i}\left(\dot{\varphi}_{0}-\dot{\varphi}_{i}\right),
$$

with

$$
\begin{aligned}
& B_{i}=m_{i} \varepsilon_{i}\left[-\left(\ddot{x}+z_{i} \ddot{\psi}_{y}\right) \sin \varphi_{i}+\left(\ddot{y}-z_{i} \ddot{\psi}_{x}\right) \cos \varphi_{i}\right], \\
& \quad i=3, \ldots, 6 .
\end{aligned}
$$

$L_{0}\left(\dot{\varphi}_{0}\right)=L_{0}^{0}-\bar{\beta}_{0} \dot{\varphi}_{0}$ is the linearized driving torque including any external damping torque. $\beta_{i}(i=$ $3, \ldots, 6)$ are the coefficients of viscosity of the two Thearle type balancers, assumed to be negligible in comparison with $\beta_{0}=\bar{\beta}_{0}+\sum_{i=3}^{6} \beta_{i} \approx \bar{\beta}_{0}$.

\section{EXISTENCE CONDITIONS FOR SYNCHRONOUS SOLUTIONS}

The system of the complete equations of motion is the mathematical model for computer simulation.

The goal of Sections 4 and 5 focuses on important approximative qualitative results with respect to existence and stability of certain useful stationary solutions under the assumption of constant angular velocity of the primary rotor

$$
\dot{\varphi}_{0}=\Omega=\text { const. }
$$

We investigate aproximately stationary solutions with equal and constant angular velocities of all compensating rotors,

$$
\dot{\varphi}_{i}=\Omega, \quad i=3, \ldots, 6 .
$$

For this task the method of direct motion separation (see Kapiza, 1951; Blekhman, 1976; Sperling et al., 1997) is well suited. Applying this method, we split the rotor angles into three components,

$$
\varphi_{i}(t)=\Omega t+\alpha_{i}(t)+\xi_{i}(t, \Omega t) ;
$$

$\alpha_{i}(t)$ is a slowly varying component. The fast component $\xi_{i}(t, \Omega t)$ is assumed to be $2 \pi$-periodic

$$
\frac{1}{2 \pi} \int_{0}^{2 \pi} \xi_{i}(t, \Omega t) \mathrm{d}(\Omega t)=0
$$


Thus, by averaging of Eq. (39) we get the equation

$$
m_{i} \varepsilon_{i}^{2} \ddot{\alpha}_{i}+\beta_{i} \dot{\alpha}_{i}+V_{i}=0
$$

for the slow component of the motion with the so-called vibrational moment as the averaged acceleration term

$$
V_{i}=\frac{1}{2 \pi} \int_{0}^{2 \pi} B_{i} \mathrm{~d}(\Omega t) .
$$

Subtraction of Eq. (45) from Eq. (39) yields the equation for the fast component,

$$
m_{i} \varepsilon_{i}^{2} \ddot{\xi}_{i}+\beta_{i} \dot{\xi}_{i}+B_{i}-V_{i}=0 .
$$

To determine the solution of the equations of motion of the axis system for stationary rotating compensating point masses, i.e. for

$$
\varphi_{i} \approx \Omega t+\alpha_{i}, \quad \alpha_{i}=\text { const., } i=3, \ldots, 6,
$$

we neglect the masses of the point masses in comparison with the mass of the basic rotor. Moreover, we confine our consideration on the undamped, isotropic case:

$$
\begin{gathered}
\boldsymbol{C}=\mathbf{0}, \\
\boldsymbol{K}=\left[\begin{array}{cccc}
k_{11} & 0 & 0 & k_{12} \\
0 & k_{11} & -k_{12} & 0 \\
0 & -k_{12} & k_{22} & 0 \\
k_{12} & 0 & 0 & k_{22}
\end{array}\right] .
\end{gathered}
$$

Thus, from Eq. (28) with expressions (29)-(36) we get, for the complex coordinates

$$
r=x+\mathrm{j} y, \quad \psi=\psi_{x}+\mathrm{j} \psi_{y}, \quad \mathrm{j}=\sqrt{-1},
$$

the equations

$$
\begin{aligned}
& {\left[\begin{array}{cc}
M & 0 \\
0 & J_{\mathrm{a}}
\end{array}\right]\left[\begin{array}{c}
\ddot{r} \\
\ddot{\psi}
\end{array}\right]+\left[\begin{array}{cc}
0 & 0 \\
0 & -\mathrm{j} J_{\mathrm{p}} \Omega
\end{array}\right]\left[\begin{array}{c}
\dot{r} \\
\dot{\psi}
\end{array}\right]} \\
& +\left[\begin{array}{cc}
k_{11} & -\mathrm{j} k_{12} \\
\mathrm{j} k_{12} & k_{22}
\end{array}\right]\left[\begin{array}{c}
r \\
\psi
\end{array}\right] \\
& \quad=\Omega^{2} \sum_{k=1}^{6} m_{k} \varepsilon_{k}\left[\begin{array}{c}
\exp \left[\mathrm{j}\left(\Omega t+\alpha_{k}\right)\right] \\
z_{k} \exp \left[\mathrm{j}\left(\Omega t+\alpha_{k}+\pi / 2\right)\right]
\end{array}\right]
\end{aligned}
$$

and the stationary solution

$$
\begin{aligned}
{\left[\begin{array}{l}
r \\
\psi
\end{array}\right]=} & \frac{\Omega^{2}}{\Delta} \sum_{k=1}^{6} m_{k} \varepsilon_{k}\left\{\left[\begin{array}{c}
\left(J_{\mathrm{p}}-J_{\mathrm{a}}\right) \Omega^{2}+k_{22} \\
-\mathrm{j} k_{12}
\end{array}\right]\right. \\
& \left.+z_{k}\left[\begin{array}{c}
\mathrm{j} k_{12} \\
-M \Omega^{2}+k_{11}
\end{array}\right] \exp \left(\mathrm{j} \frac{\pi}{2}\right)\right\} \\
& \times \exp \left[\mathrm{j}\left(\Omega t+\alpha_{k}\right)\right],
\end{aligned}
$$

with

$$
\Delta=\left|\begin{array}{cc}
-M \Omega^{2}+k_{11} & -\mathrm{j} k_{12} \\
\mathrm{j} k_{12} & \left(J_{\mathrm{p}}-J_{\mathrm{a}}\right) \Omega^{2}+k_{22}
\end{array}\right| .
$$

Notice that the second term in Eq. (52) is a gyroscopic one. The acceleration terms (40) become, under consideration of the assumption (48),

$$
\begin{aligned}
B_{i}=m_{i} \varepsilon_{i}[- & \left(\ddot{x}+z_{i} \ddot{\psi}_{y}\right) \sin \left(\Omega t+\alpha_{i}\right) \\
& \left.+\left(\ddot{y}-z_{i} \ddot{\psi}_{x}\right) \cos \left(\Omega t+\alpha_{i}\right)\right] .
\end{aligned}
$$

Equation (53) yields

$$
\begin{aligned}
& \ddot{x}+z_{i} \ddot{\psi}_{y}=-\frac{\Omega^{4}}{\Delta} \sum_{k=1}^{6} \Gamma_{i k} \cos \left(\Omega t+\alpha_{k}\right), \\
& \ddot{y}-z_{i} \ddot{\psi}_{x}=-\frac{\Omega^{4}}{\Delta} \sum_{k=1}^{6} \Gamma_{i k} \sin \left(\Omega t+\alpha_{k}\right),
\end{aligned}
$$

with the abbreviation

$$
\begin{aligned}
\Gamma_{i k}=m_{k} \varepsilon_{k}\left\{\left(J_{\mathrm{p}}-J_{\mathrm{a}}\right) \Omega^{2}+k_{22}-z_{i} k_{12}\right. \\
\left.+z_{k}\left[-k_{12}+z_{i}\left(-M \Omega^{2}+k_{11}\right)\right]\right\} .
\end{aligned}
$$

Thus, we get the acceleration terms

$$
B_{i}=m_{i} \varepsilon_{i} \frac{\Omega^{4}}{\Delta} \sum_{k=1}^{6} \Gamma_{i k} \sin \left(\alpha_{i}-\alpha_{k}\right) .
$$

This result is independent of time $t$. Therefore, this expression is identical to the vibrational moment Eq. (46) and we get the existence conditions for synchronous motions of the compensating point masses for the determination of the phase 
differences in the following simple form:

$$
V_{i}=B_{i}=0, \quad i=3, \ldots, 6 .
$$

We remark that the acceleration terms in the nonisotropic case are time dependent. Thus, the vibrational moments become different from these terms and Eq. (47) yields small fast motions with double frequency.

\section{STABILITY CONDITIONS FOR THE COMPENSATING PHASES IN THE HIGH FREQUENCY RANGE}

We assume an angular velocity in the high frequency range, i.e., we neglect all stiffness terms. Then the system has one non-vanishing natural frequency

$$
\omega_{4}=\frac{J_{\mathrm{p}}}{J_{\mathrm{a}}} \Omega .
$$

Therefore, only in the case

$$
J_{\mathrm{p}}<J_{\mathrm{a}}
$$

it is possible to make $\Omega$ greater than the highest natural frequency.

Under these conditions we get from Eqs. (58) and (59) the following system of nonlinear algebraic equations for the stationary values $\alpha_{i}^{0}(i=3, \ldots, 6)$ of the phase angles of the compensating point masses:

$$
\begin{aligned}
B_{i} & =-\frac{f_{i}}{M \Omega^{2}} \sum_{k=1}^{6} f_{k} \lambda_{i k} \sin \left(\alpha_{i}^{0}-\alpha_{k}^{0}\right)=0, \\
i & =3, \ldots, 6,
\end{aligned}
$$

with

$$
f_{i}=m_{i} \varepsilon_{i} \Omega^{2}, \quad \lambda_{i k}=1-\frac{z_{i} z_{k} M}{J_{\mathrm{p}}-J_{\mathrm{a}}}, \quad i, k=1, \ldots, 6 .
$$

However, we ask especially for the compensating solution as we are not interested in other solutions.
The conditions for perfect balance are

$$
\begin{gathered}
f_{1}+f_{2} \cos \gamma+\sum_{k=3}^{6} f_{k} \cos \alpha_{k}^{0}=0, \\
f_{2} \sin \gamma+\sum_{k=3}^{6} f_{k} \sin \alpha_{k}^{0}=0, \\
f_{1} z_{1}+f_{2} z_{2} \cos \gamma+\sum_{k=3}^{6} f_{k} z_{k} \cos \alpha_{k}^{0}=0, \\
f_{2} z_{2} \sin \gamma+\sum_{k=3}^{6} f_{k} z_{k} \sin \alpha_{k}^{0}=0 .
\end{gathered}
$$

In fact, phase angles corresponding to these conditions fulfill Eq. (62).

To analyse the stability of these solutions, we consider small perturbations of the stationary phase angles:

$$
\begin{gathered}
\alpha_{i}=\alpha_{i}^{0}+\bar{\alpha}_{i} \\
\sin \left(\alpha_{i}-\alpha_{k}\right) \approx \\
\sin \left(\alpha_{i}^{0}-\alpha_{k}^{0}\right) \\
+\cos \left(\alpha_{i}^{0}-\alpha_{k}^{0}\right)\left(\bar{\alpha}_{i}-\bar{\alpha}_{k}\right) .
\end{gathered}
$$

From the differential equations for the small perturbations,

$$
\begin{aligned}
& m_{i} \varepsilon_{i}^{2} \ddot{\bar{\alpha}}_{i}+\beta_{i} \dot{\bar{\alpha}}_{i}+\frac{1}{M \Omega^{2}} \sum_{k=3}^{6} f_{i} f_{k} \lambda_{i k} \\
& \quad \times \cos \left(\alpha_{i}^{0}-\alpha_{k}^{0}\right) \bar{\alpha}_{k}=0, \quad i=3, \ldots, 6,
\end{aligned}
$$

there follows that the motion of the compensating rotors is asymptotically stable if and only if the matrix

$$
\boldsymbol{\Lambda}=\left\{\lambda_{i k} \cos \left(\alpha_{i}^{0}-\alpha_{k}^{0}\right)\right\}
$$

is positive definite, i.e. if and only if all principal diagonal minors are positive.

The first condition

$$
\lambda_{i i}=1-\frac{z_{i}^{2} M}{J_{\mathrm{p}}-J_{\mathrm{a}}}>0
$$


shows that the motion is unstable at least under the condition

$$
J_{\mathrm{a}}<J_{\mathrm{p}}<J_{\mathrm{a}}+z_{i}^{2} M, \quad i=3, \ldots, 6 .
$$

Thus, the necessary condition (71) is fulfilled under the condition (61).

This is true also for the second necessary condition

$$
\begin{gathered}
\lambda_{i k}^{2} \sin ^{2}\left(\alpha_{i}^{0}-\alpha_{k}^{0}\right)-\frac{\left(z_{i}-z_{k}\right)^{2} M}{J_{\mathrm{p}}-J_{\mathrm{a}}}>0, \\
i, k=3, \ldots, 6, \quad i \neq k,
\end{gathered}
$$

if $\sin \left(\alpha_{i}^{0}-\alpha_{k}^{0}\right) \neq 0$.

The third and the fourth necessary conditions with the abbreviation

$$
\begin{aligned}
N_{i j k}= & 2 \cos \left(\alpha_{i}^{0}-\alpha_{j}^{0}\right) \cos \left(\alpha_{j}^{0}-\alpha_{k}^{0}\right) \cos \left(\alpha_{k}^{0}-\alpha_{i}^{0}\right) \\
& -\cos ^{2}\left(\alpha_{j}^{0}-\alpha_{k}^{0}\right)-\cos ^{2}\left(\alpha_{k}^{0}-\alpha_{i}^{0}\right)
\end{aligned}
$$

can be formulated in the following form:

$$
\lambda_{i i}\left[\lambda_{i i} \lambda_{k k} \sin ^{2}\left(\alpha_{i}^{0}-\alpha_{j}^{0}\right)+\lambda_{i k}^{2} N_{i j k}\right]>0,
$$

with $i=3, j=4, k=5$ or equivalent indices, and

$$
\begin{aligned}
& {\left[\lambda_{i i} \lambda_{k k} \sin \left(\alpha_{i}^{0}-\alpha_{j}^{0}\right) \sin \left(\alpha_{k}^{0}-\alpha_{l}^{0}\right)\right]^{2}+\lambda_{i i} \lambda_{k k} \lambda_{i k}^{2}} \\
& \quad \times\left[N_{i j k}+N_{i j l}+2 \sin ^{2}\left(\alpha_{i}^{0}-\alpha_{j}^{0}\right) \cos ^{2}\left(\alpha_{k}^{0}-\alpha_{l}^{0}\right)\right] \\
& \quad+\lambda_{i k}^{4}\left[\cos \left(\alpha_{i}^{0}-\alpha_{k}^{0}\right) \cos \left(\alpha_{j}^{0}-\alpha_{l}^{0}\right)\right. \\
& \left.\quad-\cos \left(\alpha_{i}^{0}-\alpha_{l}^{0}\right) \cos \left(\alpha_{j}^{0}-\alpha_{k}^{0}\right)\right]^{2}>0
\end{aligned}
$$

with $i=3, j=4, k=5, l=6$ or equivalent indices.

The $N$ terms are the only terms in the conditions (75) and (76) which can become negative. Therefore, it is always possible to guarantee by suitable choice of the values of $z_{3}$ and $z_{5}$, that $\lambda_{i k}, i=3, k=5$, is sufficiently small and that the conditions (75) and (76) are fulfilled, if condition (61) holds. For instance, numerical calculations for the parameter sets of the computer simulation reported in the following and for the ones in Heydaya and Sharp (1977) result always in positive definite matrices (70) if condition (61) is fulfilled.

In the case of too great values of the polar inertia the generalized self-balancing principle, formulated by Blekhman (1993) and applicable for a great number of systems, but without a general proof, is obviously not valid, because the angular velocity of the rotor is not higher than the maximum natural frequency in consequence of the influence of the gyroscopic terms of the equations of motion of the primary rotor.

The above stability consideration was restricted to the equations of motion of the compensating rotors. In general the equations of motion of the primary rotor must be considered, too. But, Hedaya and Sharp (1997) found that "the rotor modes are a little dependent on the balancer characteristics and can be substantially understood in terms of the behaviour of the rotor alone". Therefore, in this paper we confirm our results concerning the stability of the primary rotor motion only by computer simulation.

\section{RESULTS OF COMPUTER SIMULATION}

The aim of the computer simulations is to confirm the analytical approximate results. Compared to the analytical solution the numerical simulation offers some additional features, such as investigation of transient motions, consideration of the complete equations of motion including oscillator damping. Simulations are a suitable tool to complement the analytical stability investigations.

The simulations were carried out by means of the simulation language ACSL. The DASSL code was used as an algorithm to integrate the complete set of the equations of motion (28)-(40). The course of motion presented in Fig. 4 is the result of simulation with the following dimensionless 

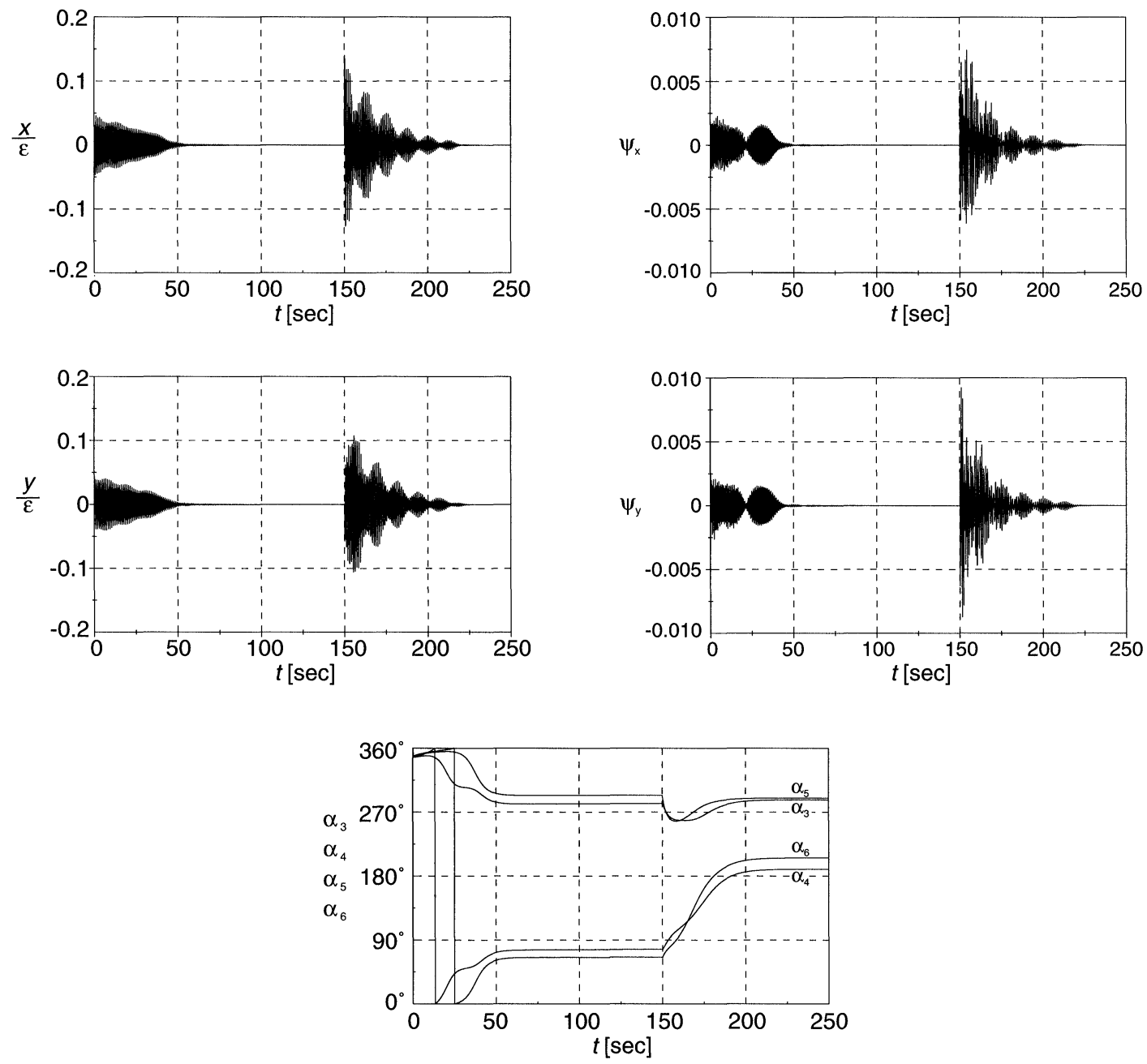

FIGURE 4 Automatic balancing after start-up of the primary rotor.

parameters (with $\boldsymbol{C}$ corresponding to $\boldsymbol{K}$, Eq. (50)):

$$
\begin{aligned}
\frac{J_{\mathrm{a}}}{M \varepsilon^{2}} & =33.84 ; \quad \frac{k_{11}}{M \Omega^{2}}=4.44 \cdot 10^{-2} ; \\
\frac{k_{12}}{M \Omega^{2} \varepsilon} & =0.111 ; \quad \frac{k_{22}}{M \Omega^{2} \varepsilon^{2}}=4.44 ; \\
\frac{J_{\mathrm{p}}}{M \varepsilon^{2}} & =10.00 ; \quad \frac{c_{11}}{M \Omega}=3.33 \cdot 10^{-3} ; \\
\frac{c_{12}}{M \Omega \varepsilon} & =8.33 \cdot 10^{-3} ; \quad \frac{c_{22}}{M \Omega \varepsilon^{2}}=0.333 ; \\
\frac{m_{1}}{M}=\frac{m_{2}}{M} & =0.01 ; \quad \frac{m_{i}}{M}=0.008, \quad i=3, \ldots, 6 ;
\end{aligned}
$$

$$
\begin{gathered}
\frac{\varepsilon_{i}}{\varepsilon}=1, \quad i=1,3, \ldots, 6 ; \quad \frac{\varepsilon_{2}}{\varepsilon}=2 ; \quad \gamma=\pi ; \\
\frac{z_{1}}{\varepsilon}=-1 ; \quad \frac{z_{2}}{\varepsilon}=1 ; \quad \frac{z_{3}}{\varepsilon}=\frac{z_{4}}{\varepsilon}=-10 ; \\
\frac{z_{5}}{\varepsilon}=\frac{z_{6}}{\varepsilon}=10 ; \quad \frac{\beta_{i}}{M \Omega \varepsilon^{2}}=3.33 \cdot 10^{-2}, \quad i=3, \ldots, 6,
\end{gathered}
$$

with the reference values $M=1 \mathrm{~kg}, \varepsilon=0.01 \mathrm{~m}$ and $\Omega=30 \mathrm{~s}^{-1}$.

Figure 4 shows the automatic balancing after the start-up of the primary rotor, where the characteristic of the drive was modelled by a nonlinear characteristic in contradistinction to Eq. (37). 
The initial conditions of the simulation correspond to the resting system, i.e. all state variables and their derivatives are equal to zero at $t=0$.

With the chosen parameters of the drive the angular velocity of the rotor reaches very quickly $(t \approx 1 \mathrm{~s})$ the value $\Omega$, which is almost three times the highest natural frequency of the non-rotating oscillator. After that the angular velocity of the rotor is nearly constant $\left(\dot{\varphi}_{0} \approx \Omega\right)$. In the synchronous balanced state the oscillations have nearly vanished. At $t=150 \mathrm{~s}$ the angle $\gamma$ has changed to $\pi / 2$ and after a transient motion a new stable stationary motion is established with the system balanced by the synchronized rotor motions. The magnitudes of the stationary solutions in the case of excitation only by the unbalance of the primary rotor, i.e. for the system without compensating point masses are, for $\gamma=\pi$,

$$
\begin{aligned}
& \frac{x_{\max }}{\varepsilon}=\frac{y_{\max }}{\varepsilon} \approx 1.0 \cdot 10^{-2}, \\
& \psi_{x_{\max }}=\psi_{y_{\max }} \approx 1.4 \cdot 10^{-3},
\end{aligned}
$$

and for $\gamma=\pi / 2$

$$
\begin{aligned}
& \frac{x_{\max }}{\varepsilon}=\frac{y_{\max }}{\varepsilon} \approx 2.2 \cdot 10^{-2}, \\
& \psi_{x_{\max }}=\psi_{y_{\max }} \approx 1.0 \cdot 10^{-3} .
\end{aligned}
$$

Systematic simulation studies showed that condition (61) is a necessary condition for stable synchronous motion of the balanced system; however, wether this state is reached after a transient motion depends also on the coefficients of viscosity $\beta_{i}$ of the compensation rotors $(i=3, \ldots, 6)$. If the values $\beta_{i}$ are too small, no transition to a synchronous motion can be observed after the start-up of the primary rotor, although $J_{\mathrm{p}}$ is considerably smaller than $J_{\mathrm{a}}$. If the values $\beta_{i}$ become too large, the time of transient motion increases significantly, which means that not only condition (61) must be fulfilled but also $\beta_{i}$ must have appropriate values to get automatic balancing of the system investigated. If no synchronous motion is achieved due to small $\beta_{i}$ the angular velocities of the compensation rotors do not reach $\Omega$. Hence the system is not synchronized and the exiting forces and moments are not compensated.
Starting from a stationary synchronous motion it was possible to find cases of synchronous motion with $0.9 J_{\mathrm{a}}<J_{\mathrm{p}}<J_{\mathrm{a}}$. Switching $J_{\mathrm{p}}$ from a small value to a value nearly equal to $J_{\mathrm{a}}$ disturbs the stationary motion slightly. In this case the system remains synchronized and balanced if $J_{\mathrm{p}}<J_{\mathrm{a}}$, but if $J_{\mathrm{p}}>J_{\mathrm{a}}$ the system falls out of the synchronized and balanced state.

We also carried out simulations with parameters according to Hedaya and Sharp (1997), where always $J_{\mathrm{p}}<J_{\mathrm{a}}$. In our simulations all cases result in synchronous and balanced motions if the values $\beta_{i}$ are chosen appropriately, even the case with the largest $J_{\mathrm{p}}$, which is unstable according to the stability analysis in Hedaya and Sharp (1997).

\section{FINAL REMARKS}

Further investigations on the known principle of automatic balancing of a statically and dynamically unbalanced rigid rotor by two pairs of compensating balancers, freely rotating in a viscous medium (see Hedaya and Sharp, 1997), are presented. The method of direct motion separation is proved as well suited for the development of existence and stability conditions for synchronous compensating motion. In the high frequency range such motions are possible under certain conditions. The compensating motion is stable if and only if a special $4 \times 4$ matrix is positive definite. This matrix depends in a simple manner on the compensating phases, on the mass, on the polar and the transverse inertia of the basic rotor, and on the axial distances between compensating balancers and the mass centre of the primary rotor. Especially the explicit necessary stability condition is found, viz. that the polar inertia of the primary rotor must be smaller than the transverse inertia. Otherwise the requirements of Blekhman's generalized self-balancing principle are not met.

These results are confirmed by computer simulation. However, the closer the polar inertia are to the transverse one, the greater the sensitivity of the compensating motion with respect to the coefficients of the viscosity of the balancers and to the initial conditions. 
Thus, the application of the method in practice is more restricted than a force balancer employed, for instance, in centrifuges. At present we observe a revival of applications of the automatic balancing principle in practice. SKF developed and manufactured a balancing unit, called Auto-Balancing, that automatically compensates for the imbalance during operation. There are many potential applications for this unit, for instance in hand-held power tools and in washing machines. Atlas Copco manufactured a new hand-held grinder fitted with this unit. ETI-Canada has the European Patent EP 0640192 B1 "An Unbalance Compensating Method and Apparatus" with the priority from 21 May 1992. Recently the first washing machine with an automatic balancing system was put on the market by Samsung.

Therefore, it seems to be very important and useful to do further research with respect to the robustness of the compensating motion in the case of two force balancers and with respect to improvements towards applications in practice.

\section{Acknowledgement}

Authors would like to acknowledge the Deutsche Forschungsgemeinschaft for financial support (No. Sp 462/2-1/2).

\section{NOMENCLATURE}

$B, B_{i}$
$\boldsymbol{C}$
$\overrightarrow{\boldsymbol{e}}_{x}, \overrightarrow{\boldsymbol{e}}_{y}, \overrightarrow{\boldsymbol{e}}_{z}$
$f_{i}$
$\mathrm{j}$
$\boldsymbol{J}$
$J_{\mathrm{a}}$
$J_{\mathrm{p}}$
$\boldsymbol{K}$
$L_{0}$
$M, m_{i}$
$N_{i j k}$
$\boldsymbol{q}$

abbreviations, Eqs. (26), (38), (40) damping matrix with elements $c_{i j}$ vector base centrifugal force imaginary unity inertia matrix with elements $J_{i j}$ moment of transverse inertia moment of polar inertia stiffness matrix with elements $k_{i j}$ driving torque of the primary rotor mass abbreviation, Eq. (74) vector of generalized coordinates $\left[x ; y ; \psi_{x} ; \psi_{y}\right]^{\mathrm{T}}$

\begin{tabular}{|c|c|}
\hline$\underset{r}{\boldsymbol{Q}}, \boldsymbol{Q}_{\mathrm{total}}, \boldsymbol{Q}_{i}$ & $\begin{array}{l}\text { acceleration terms } \\
\text { complex coordinate } x+\mathrm{j} y\end{array}$ \\
\hline$t$ & time \\
\hline$V_{i}$ & vibrational moment \\
\hline$z_{i}$ & distance in axial direction \\
\hline$\alpha_{i}$ & $\begin{array}{l}\text { slowly varying component } \\
\text { of rotor angle }\end{array}$ \\
\hline$\beta_{i}$ & $\begin{array}{l}\text { damping coefficient of rotational } \\
\text { motion }\end{array}$ \\
\hline$\Gamma_{i k}$ & abbreviation, Eq. (57) \\
\hline$\Delta$ & abbreviation, Eq. (54) \\
\hline$\varepsilon_{i}$ & eccentricity \\
\hline$\lambda_{i k}$ & abbreviation, $1-\left(z_{i} z_{k} M\right) /\left(J_{\mathrm{p}}-J_{\mathrm{a}}\right)$ \\
\hline$\Lambda$ & abbreviation, $\left\{\lambda_{i k} \cos \left(\alpha_{i}^{0}-\alpha_{k}^{0}\right)\right\}$ \\
\hline$\xi_{i}$ & small fast component of rotor angle \\
\hline$\varphi_{i}$ & rotor angle \\
\hline$\psi$ & complex coordinate $\psi_{x}+\mathrm{j} \psi_{y}$ \\
\hline$\omega_{4}$ & natural frequency \\
\hline$\Omega$ & angular velocity \\
\hline
\end{tabular}

\section{References}

Thearle, E.L. and Schenectady, N.Y. (1932) A new type of dynamic-balancing machine, Trans. A.S.M.E. 54(12) $131-141$

Hedaya, M.T. and Sharp, R.S. (1977) An analysis of a new type of automatic balancer, Journal Mechanical Engineering Science, 19(5), 221-226.

Sperling, L., Duckstein, H. and Merten, F. (1997) Synchrone Entstörung von Schwingungen infolge unwuchtiger Rotoren, In Irretier, H., Nordmann, R. and Springer, H. (Eds.) Schwingungen in Rotierenden Maschinen IV, Vieweg, 309316

Sperling, L. (1994) Selbstsynchronisation statisch und dynamisch unwuchtiger Vibratoren, Teil I: Grundlagen, Technische Mechanik 14(1), 61-76.

Sperling, L. (1994) Selbstsynchronisation statisch und dynamisch unwuchtiger Vibratoren, Teil II: Ausführung und Beispiele, Technische Mechanik 14(2), 85-96.

Blekhman, I.I. (1988) Synchronization in Science and Technology, ASME TRANSLATIONS, New York.

Blekhman, I.I. (1993) The generalized self-balancing principle, Proc. of Asia-Pacific Vibration Conference 93(2), Kitakyshu, Japan, 509-514.

Kapiza, P.L. (1951) Dynamic stability of a pendulum with vibrating support point, Journal Experimental and Theoretical Physics 21(5), 588-598.

Blekhman, I.I. (1976) Method of direct motion separation in problems of vibration acting on nonlinear mechanical systems, Izv. AN SSSR Mekkanika Tverdogo Tela 11(6), $13-27$.

Sperling, L., Merten, F. and Duckstein, H. (1997) Rotation und Vibration in Beispielen zur Methode der direkten Bewegungsteilung, Technische Mechanik 17(3), 231-243. 


\section{ait \\ ENERGY MATERIALS}

M A N E Y publishing

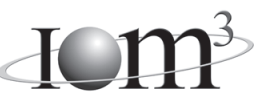

\section{Materials Science \& Engineering for Energy Systems}

Maney Publishing on behalf of the Institute of Materials, Minerals and Mining

The Institute of Materials, Minerals \& Mining

Economic and environmental factors are creating ever greater pressures for the efficient generation, transmission and use of energy. Materials developments are crucial to progress in all these areas: to innovation in design; to extending lifetime and maintenance intervals; and to successful operation in more demanding environments. Drawing together the broad community with interests in these areas, Energy Materials addresses materials needs in future energy generation, transmission, utilisation, conservation and storage. The journal covers thermal generation and gas turbines; renewable power (wind, wave, tidal, hydro, solar and geothermal); fuel cells (low and high temperature); materials issues relevant to biomass and biotechnology; nuclear power generation (fission and fusion); hydrogen generation and storage in the context of the 'hydrogen economy'; and the transmission and storage of the energy produced.

As well as publishing high-quality peer-reviewed research, Energy Materials promotes discussion of issues common to all sectors, through commissioned reviews and commentaries. The journal includes coverage of energy economics and policy, and broader social issues, since the political and legislative context influence research and investment decisions.

\section{CALL FOR PAPERS}

Contributions to the journal should be submitted online at http://ema.edmgr.com

To view the Notes for Contributors please visit: www.maney.co.uk/journals/notes/ema

Upon publication in 2006, this journal will be available via the Ingenta Connect journals service. To view free sample content online visit: www.ingentaconnect.com/content/maney

For further information please contact:

Maney Publishing UK

Tel: +44 (0)113 2497481 Fax: +44 (0)1132486983 Email: subscriptions@maney.co.uk

or

Maney Publishing North America

Tel (toll free): 8662975154 Fax: 6173546875 Email: maney@maneyusa.com

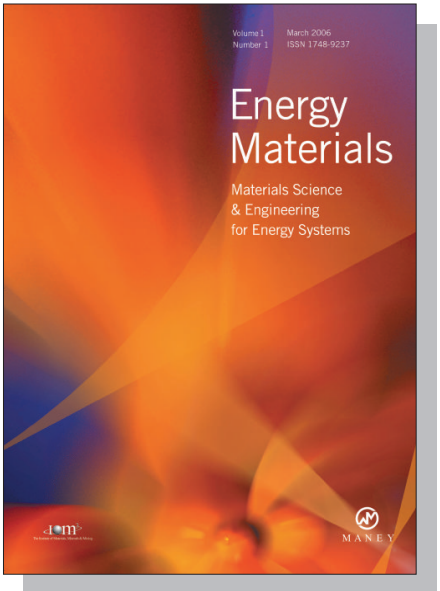

EDITORS

Dr Fujio Abe

NIMS, Japan

Dr John Hald, IPL-MPT, Technical University of Denmark, Denmark

Dr R Viswanathan, EPRI, USA

\section{SUBSCRIPTION INFORMATION}

Volume 1 (2006), 4 issues per year

Print ISSN: 1748-9237 Online ISSN: 1748-9245

Individual rate: $£ 76.00 / U S \$ 141.00$

Institutional rate: $£ 235.00 /$ US $\$ 435.00$

Online-only institutional rate: $£ 199.00 / U S \$ 367.00$

For special $\mathrm{IOM}^{3}$ member rates please email

subscriptions@maney.co.uk

\section{For further information or to subscribe online please visit www.maney.co.uk}



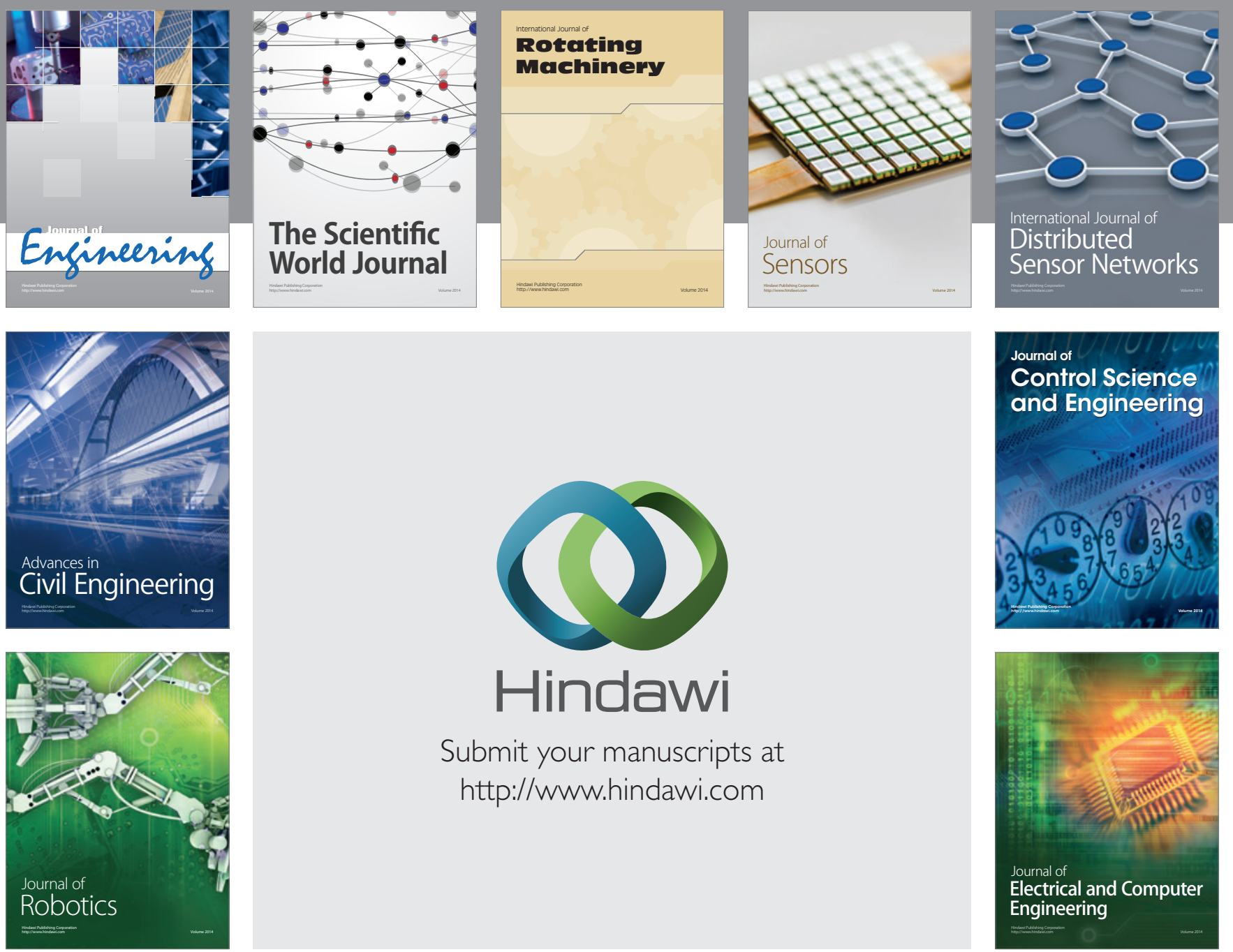

Submit your manuscripts at

http://www.hindawi.com
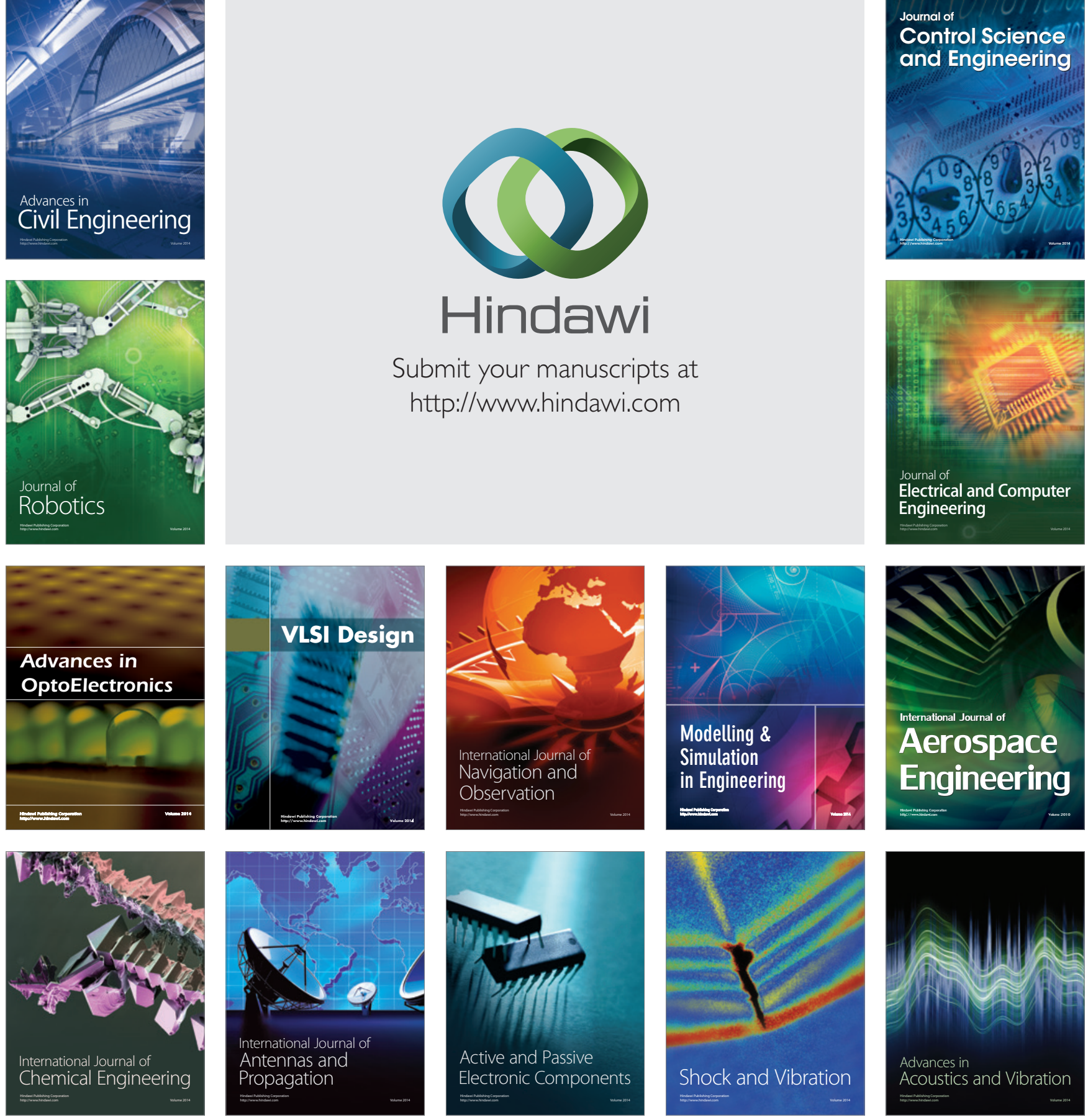